\title{
A preliminary list of the Fanniidae and Muscidae (Diptera) of Belarus
}

\section{Предварительный список Fanniidae и Muscidae (Diptera) Беморуссии}

\author{
E.V. Makovetskaya ${ }^{1}$, N.E. Vikhrev ${ }^{2}$ \\ Е.В. Маковецкая ${ }^{1}$, Н.Е. Вихрев ${ }^{2}$
}

\footnotetext{
${ }^{1}$ The Scientific and Practical Center for Bioresources, Akademicheskaya 27, Minsk 220072, Belarus. E-mail: makovetskayaev@tut.by

${ }^{1}$ ГНПО «НПЦ НАН Беларуси по биоресурсам», Академическая ул. 27, Минск 220072, Беларусь.

${ }^{2}$ Zoological Museum of Moscow University, Bolshaya Nikitskaya 2, Moscow 125009, Russia. E-mail: nikita6510@ya.ru

2 Зоологический музей, Московский государственный университет им. М.В. Ломоносова, Большая Никитская ул. 2, Москва 125009 , Россия.
}

KEY WORDS: fauna, Belarus, Fanniidae, Muscidae.

КЛЮЧЕВЫЕ СЛОВА: фауна, Белоруссия, Fanniidae, Muscidae.

ABSTRACT. A preliminary list of the Fanniidae and Muscidae of Belarus, consisting of 124 species, is provided: 22 species, that are 6 species of Fanniidae and 16 of Muscidae, are indicated for Belarus for the first time.

РЕЗЮМЕ. Приведён предварительный список Fanniidae и Muscidae Беларуси, содержащий 124 вида. Впервые для Беларуси указаны 22 вида двукрылых: 6 видов Fanniidae и 16 - Muscidae.

\section{Introduction}

The fauna of Belarus is still badly known. During the Soviet epoch, there were a lot of more interesting places than the Belarusian lowlands: the Caucasus; the mountains, steppes and deserts of the Central Asia; the Far East; Arctic regions.

The entomological collections created during the Russian Empire were destroyed during the Second World War. In the post-Soviet time, Belarus did not resume to the study of the Calyptratae until present. However, the fauna of Belarus is very interesting for Russian entomologists because of the presence of West European species, and for European entomologists because of the presence of East Palaearctic species. There is a unique locality in Belarus: the famous Bialowieza Forest or Belovezhskaya Pushcha, which is one of the last and largest remaining parts of the immense primeval forest that once stretched across the European Plain.

In the XIX century the Muscidae of Belarus was studied by Portschinsky [1874, 1880, 1892], Schnabl [1877, 1881, 1886, 1887a, b, 1888] and Arnold [1901].
Later the Belarusian fauna of Muscidae and Fanniidae was considered in little known publications of Birg [1971]; in the Catalogue of Palaearctic Diptera [Pont, 1986a, b] and in Draber-Monko [1995, 2001]. The data on the European Fanniidae were summarized in Rozkošný et al. [1997]. Some new records of Belarusian Muscidae were published in Vikhrev [2014, 2015a, b, 2018], Vikhrev \& Erofeeva [2018].

The XXI century added a new resource: the web database Fauna Europaea [Pont, 2013], and we had a long discussion about whether to use it or not. The arguments against were as follows: it is not an appropriate source of scientific data (1); it gives no information about where specimens were collected or are stored (2). But there were strong arguments for using this source. Traditional publications, for example, the Catalogue of Palaearctic Diptera also may omit label information. The taxonomic specialist in Muscidae and Fanniidae in the Fauna Europaea project is Dr. Adrian C. Pont, who is a well known expert in these groups, so their identifications are as reliable as possible.

Birg [1971] published her report on synanthropic flies of Belarus being a postgraduate student with a limited experience in identification.

Since in that paper the label data are absent in many cases and identifications may be doubtful in some cases, we will give our records for previously recorded species too.

The publication of Draber-Monko [2001] created another problem. She investigated the fauna of Belovezhskaya Pushcha on either Belarusian and Polish sides of the forest. The most remote Polish localities are in $20 \mathrm{~km}$ from Polish-Belarusian border. About the

How to cite this article: Makovetskaya E.V., Vikhrev N.E. 2019. A preliminary list of the Fanniidae and Muscidae (Diptera) of Belarus // Russian Entomol. J. Vol.28. No.1. P.93-101. doi: 10.15298/rusentj.28.1.17 


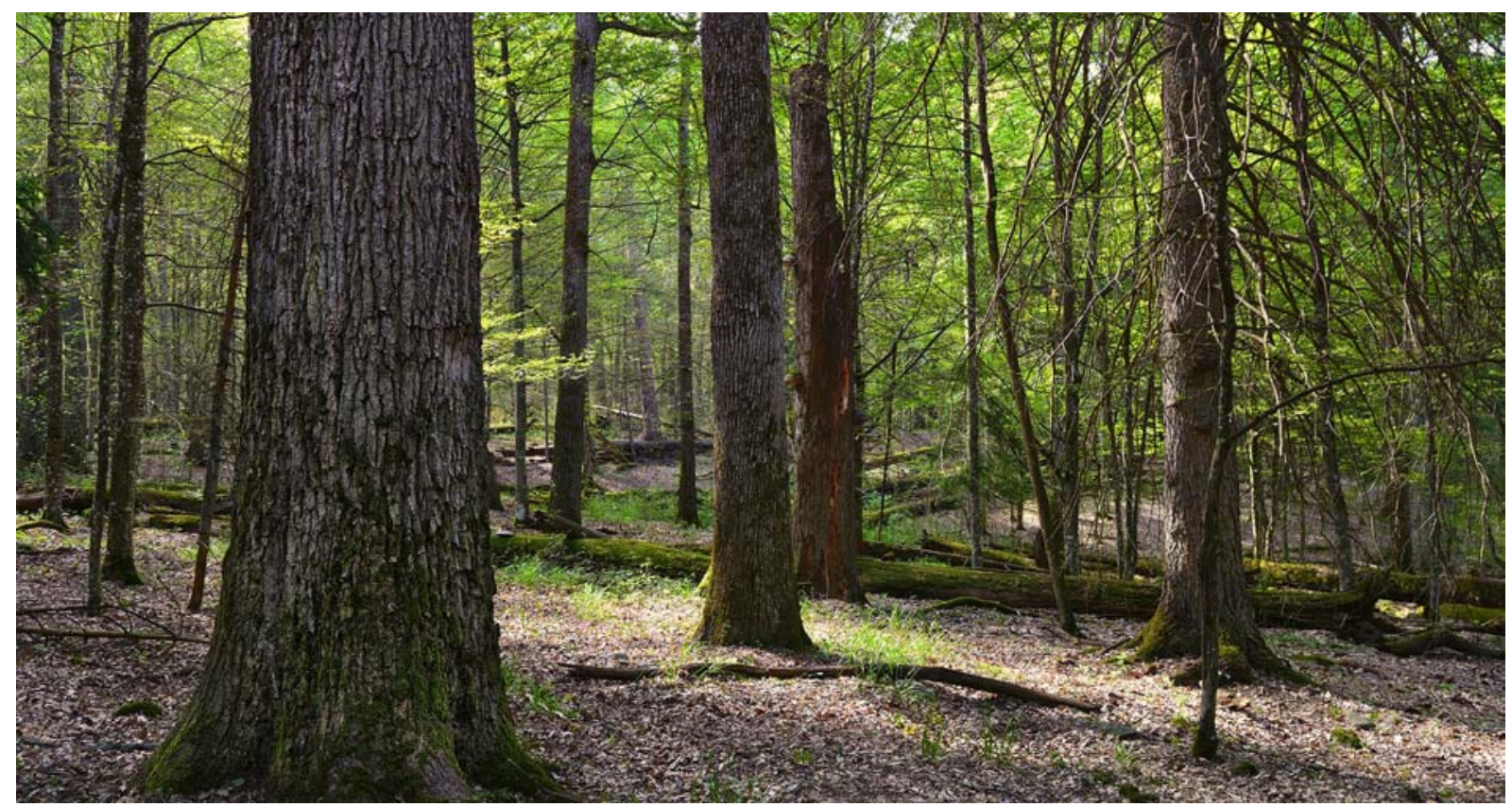

Fig. 1. Belovezhskaya Pushcha (photo: O. Prishchepchik).

Рис. 1. Беловежская Пуща (фото: О. Прищепчик)

Muscidae and Fanniidae fauna, Draber-Monko indicated records as made from Polish part, and cited Birg [1971] for the Belarusian part. Formally we should consider only Belarusian records, so we did. On the other hand, Belovezhskaya Pushcha is a single and medium large $\left(3000 \mathrm{~km}^{2}\right)$ forest, so there is no reason to divide it by political border, which is biogeographically artificial. So, we decided to list the Bialowiezian species recorded only from Polish part of the forest in special Addendum to this paper.

Before this work, 8 species of Fanniidae and 94 species of Muscidae were known for the fauna of Belarus, 22 more species of dipterans (6 Fanniidae and 16 Muscidae) are indicated here for Belarus for the first time.

\section{Material and methods}

The classification follows that in Pont [1986] but also includes more recent changes. Synonyms are only given for names that are used in the faunistic references cited in the paper. Suprageneric taxa (subfamilies and tribes) are arranged in systematic order. Names of genera are arranged as in Pont [1986], and species are listed alphabetically within each genus.

Geographical coordinates are given in the decimal degrees format.

The specimens examined in this study are deposited in the following institutions:

ZMUM - Zoological Museum of Moscow University, Russia;

ZIN - Zoological Institute, St-Petersburg, Russia;

SPCB - The Scientific and Practical Center for Bioresources, Minsk, Belarus.
A preliminary list of the Fanniidae and Muscidae (Diptera) of Belarus

Family Fanniidae

1. Fannia armata (Meigen, 1826)

Vikhrev [2018]: Vitebsk reg.: Orsha distr., Dnieper R., $54.543^{\circ} \mathrm{N} 30.463^{\circ} \mathrm{E}, 11$. VI.2017.

2. Fannia canicularis (Linnaeus, 1761)

Birg [1971]: widespread. Also Arnold [1901] as Homalomyia canicularis (Linnaeus, 1761), Danilevich [1958], Erofeeva et al. [1960], Gembicky [1968], Neselovskaya et al. [1968], Nakhaenko, Kaplich [2007].

MATERIAL: Minsk reg.: Minsk, Akademicheskaya str., $53.916^{\circ} \mathrm{N} 27.603^{\circ} \mathrm{E}, 25 . I X .2018$, E. Makovetskaya, $10^{7}$ (SPCB). Vitebsk reg.: Polotsk distr., Rudnya env. $\left(\approx 55.45^{\circ} \mathrm{N} 28.57^{\circ} \mathrm{E}\right)$, 31.VII.1965, A.V. Birg, $10^{7}$ (SPCB).

3. Fannia carbonaria (Meigen, 1826)

Birg [1971]: Belovezhskaya Pushcha.

4. Fannia corvina (Verrall, 1892)

MATERIAL: Vitebsk reg.: Orsha distr., Dnieper R., $54.543^{\circ} \mathrm{N}$ $30.463^{\circ} \mathrm{E}, 11 . \mathrm{VI} .2017$, N. Vikhrev, $20^{\top} \mathrm{O}^{\top} 1$ 1ㅇ (ZMUM).

5. Fannia fuscula (Fallen, 1825)

MATERIAL: Minsk reg.: Barysaw env., $54.256^{\circ} \mathrm{N} 28.481^{\circ} \mathrm{E}$, 05.VII.2013, D. Gavryushin, $10^{7}$ (ZMUM). Vitebsk reg.: Orsha distr., Dnieper R., $54.543^{\circ} \mathrm{N} 30.463^{\circ} \mathrm{E}, 11 . \mathrm{VI} .2017$, N. Vikhrev, $10^{7}$ (ZMUM).

\section{Fannia incisurata (Zetterstedt, 1838)}

Birg [1971]: Minsk and Vitebsk reg., Dokshytsy distr.

7. Fannia lustrator (Harris, 1780)

Pont [1986a, 2013]; Rozkošný et al. [1997].

MATERIAL: Brest reg.: border of Kobryn and Pruzhany dis- 
tricts, near Oranchitsy vill., $52.398^{\circ} \mathrm{N} 24.565^{\circ} \mathrm{E}, 16-29 . \mathrm{VII} .2017$, E. Makovetskaya, $1+$ (SPCB). Vitebsk reg.: Orsha distr., Dnieper R., $54.543^{\circ} \mathrm{N} 30.463^{\circ} \mathrm{E}, 11$. VI.2017, N. Vikhrev, $10^{7}$ (ZMUM).

\section{Fannia metallipennis (Zetterstedt, 1838)}

Wang et al. [2013].

REMARKS. The mention of Belarus in Wang et al. [2013] is most probably an error. The authors wrote that they worked with Chinese material only, so they could get Belarusian record only from literature, but there are no other mentions of the presence of $F$. metallipennis in Belarus.

\section{Fannia polychaeta (Stein, 1895)}

MATERIAL: Minsk reg.: Barysaw env., $54.256^{\circ} \mathrm{N} 28.481^{\circ} \mathrm{E}$, 05.VII.2013, D. Gavryushin, $10^{7}$ (ZMUM).

\section{Fannia scalaris (Fabricius, 1794)}

Erofeeva et al. [1960]: Minsk; Birg, [1971]: Belovezhskaya Pushcha, Minsk and Vitebsk reg.

11. Fannia serena (Fallen, 1825)

Pont [1986a, 2013]; Rozkošný et al. [1997].

12. Fannia similis (Stein, 1895)

MATERIAL: Minsk reg.: Barysaw env., $54.256^{\circ} \mathrm{N} 28.481^{\circ} \mathrm{E}$ 05.VII.2013, D. Gavryushin, $10^{\top}$ (ZMUM). Vitebsk reg.: Orsha distr., Dnieper R., $54.543^{\circ} \mathrm{N} 30.463^{\circ} \mathrm{E}, 11$. VI.2017, N. Vikhrev, $2 \sigma^{7} \sigma^{7}$ (ZMUM); Orsha distr., Svyatoe L., $54.686^{\circ} \mathrm{N} 30.442^{\circ} \mathrm{E}$, 11.VI.2017, N. Vikhrev, $10^{7}$ (ZMUM).

\section{Fannia sociella (Zetterstedt, 1845)}

MATERIAL: Minsk reg.: Barysaw env., $54.256^{\circ} \mathrm{N} 28.481^{\circ} \mathrm{E}$, 05.VII.2013, D. Gavryushin, $10^{7}$ (ZMUM).

\section{Fannia spathiophora Malloch, 1918}

MATERIAL: Vitebsk reg.: Orsha distr., Dnieper R., $54.543^{\circ} \mathrm{N}$ 30.463 ${ }^{\circ} \mathrm{E}, 11$. VI.2017, N. Vikhrev, $10^{7}$ (ZMUM).

\section{Family Muscidae}

Subfamily Muscinae

\section{Muscina levida (Harris, 1780)}

Birg [1971]: as M. assimilis (Fallen, 1823), widespread. Also Erofeeva et al. [1960], Draber-Monko [1995].

MATERIAL: Brest reg.: Belovezhskaya Pushcha, $52.573^{\circ} \mathrm{N}$ 23.802 ${ }^{\circ}$ E, 06.X.2017, E. Makovetskaya, 1 ( (SPCB). Minsk reg.: Barysaw distr., near Veselovo vill., $54.382^{\circ} \mathrm{N} 28.343^{\circ} \mathrm{E}, 14 . \mathrm{VI} .2017$, E. Makovetskaya, $10^{7}$ (SPCB); Lahoysk distr., Malinovka vill., $54.466^{\circ} \mathrm{N} 27.961^{\circ} \mathrm{E}, 18 . \mathrm{VI} .2017$, E. Makovetskaya, 1 을 (SPCB) Minsk, Central Botanical Garden, $53.911^{\circ} \mathrm{N} 27.619^{\circ} \mathrm{E}, 26 . \mathrm{VII} .2017$, E. Makovetskaya, $10^{7}$ (SPCB); Minsk, Soltysa str., $53.896^{\circ} \mathrm{N}$ $27.657^{\circ} \mathrm{E}, 28$. VI.2017, E. Makovetskaya, $8 \sigma^{7} \sigma^{7}$ (SPCB); Minsk distr., near Volchkovichy, 53.809 ${ }^{\circ} \mathrm{N} 27.384^{\circ} \mathrm{E}, 15 . \mathrm{VI} .2017$, E. Makovetskaya, $10^{7}$ (SPCB); Stolbcy distr., $53.508^{\circ} \mathrm{N} 26.665^{\circ} \mathrm{E}$ 10.VI.2018, E. Makovetskaya, 1 ㅇ (SPCB); Stolbcy distr., near Semenchitsy, $53.545^{\circ} \mathrm{N} 26.699^{\circ} \mathrm{E}, 20 . \mathrm{VIII} .2018$, E. Makovetskaya, $1 \sigma^{7}$ (SPCB); Volozhin distr., Nalybokskaya Pushcha, Belokorets vill., $54.018^{\circ} \mathrm{N} 26.509^{\circ} \mathrm{E}$, 17.IX.2017, E. Makovetskaya, $10^{7}$ (SPCB. Vitebsk reg.: Polotsk distr., Rudnya env. ( $\left.\approx 55.45^{\circ} \mathrm{N} 28.57^{\circ} \mathrm{E}\right)$, 31.VII.1965, A.V. Birg, $20^{7} \sigma^{7}$ (SPCB).

\section{Muscina pasquorum (Meigen, 1826)}

Erofeeva et al. [1960]: Minsk; Birg [1971]: Minsk, Brest and Belovezhskaya Pushcha; Draber-Monko [1995]: Berezinsky Biosphere Reserve.

MATERIAL: Minsk reg.: Volozhin distr., Nalybokskaya Pushcha, Belokorets vill., 54.018 ${ }^{\circ} \mathrm{N} 26.509^{\circ} \mathrm{E}, 17 . I X .2017$, E. Makovetskaya, $10^{7}$ (SPCB).

\section{Muscina prolapsa (Harris, 1780)}

Erofeeva et al. [1960]: Minsk; Birg [1971]: Belovezhskaya Pushcha; Draber-Monko [1995]; Berezinsky Biosphere Reserve.

MATERIAL: Minsk reg.: Minsk, Central Botanical Garden, $53.911^{\circ} \mathrm{N} 27.619^{\circ} \mathrm{E}, \mathrm{III}-\mathrm{IV} .2017$, E. Makovetskaya, $10^{7}$ (SPCB); Minsk distr., near Volchkovichy vill., $53.809^{\circ} \mathrm{N} 27.384^{\circ} \mathrm{E}, 15 . \mathrm{VI}$. 2017, E. Makovetskaya, 1 우 (SPCB).

\section{Muscina stabulans (Fallen, 1817)}

Birg [1971]: widespread. Also Salaview [1930], Danilevich [1958], Erofeeva et al. [1960], Neselovskaya et al. [1968].

MATERIAL: Minsk reg.: Lahoysk distr., Malinovka vill., $54.466^{\circ} \mathrm{N} 27.961^{\circ} \mathrm{E}, 18 . \mathrm{VI} .2017$, E. Makovetskaya, $2 \sigma^{\top} \sigma^{\top} 1$ 우 (SPCB); Stolbcy distr., near Semenchitsy, $53.545^{\circ} \mathrm{N} 26.699^{\circ} \mathrm{E}$, 20.VIII.2018, E. Makovetskaya, $2 \sigma^{7} \sigma^{7}$ (SPCB); Volozhin distr., Kapustino env. $\left(\approx 54.06^{\circ} \mathrm{N} 26.55^{\circ} \mathrm{E}\right), 14$.VII.1965, A.V. Birg, $10^{7}$ (SPCB). Vitebsk reg.: Senno distr., Kruglyany env. $\left(\approx 54.89^{\circ} \mathrm{N}\right.$ 29.98 ${ }^{\circ}$ E), 25-26.V.1966, A.V. Birg, $10^{7}$ (SPCB).

\section{Azelia aterrima Meigen, 1826}

Schnabl [1877, 1881]: Minsk province. Also Pont [2013].

\section{Azelia cilipes (Haliday, 1838)}

Vikhrev [2015a]: Minsk reg., Barysaw env.

MATERIAL: Minsk reg.: Barysaw env., $54.256^{\circ} \mathrm{N} 28.481^{\circ} \mathrm{E}$, 5.VII.2013, D. Gavryushin, $10^{7}$ (ZMUM). Vitebsk reg.: Orsha distr., Dnieper R., $54.543^{\circ} \mathrm{N} 30.463^{\circ} \mathrm{E}, 11 . \mathrm{VI} .2017$, N. Vikhrev, $20^{7} \sigma^{7}$ (ZMUM).

\section{Azelia zetterstedtii Rondani, 1866}

Schnabl [1877, 1881]: Minsk province. Also Pont [2013].

\section{Thricops cunctans (Meigen, 1826)}

Schnabl [1886, 1887a]: as Aricia hirsutula (Zetterstedt, 1837) Minsk reg., Pukhovichy distr. (Minsk province), and Vitebsk reg., Liozno distr. (Mogilev province). Also Pont [1986b, 2013].

\section{Thricops diaphanus (Wiedemann, 1817)}

Schnabl [1886, 1887a]: as Aricia aculeata Loew, 1873, Minsk reg., Pukhovichy distr. (Minsk province), and Vitebsk reg., Liozno distr. (Mogilev province). Also Pont [1986b, 2013].

\section{Thricops longipes (Zetterstedt, 1845)}

Schnabl [1886, 1887a]: as Aricia longipes (Zetterstedt, 1845), Vitebsk reg., Liozno distr. (Mogilev province). Also Pont [2013].

\section{Thricops nigrifrons (Robineau-Desvoidy, 1830)}

Schnabl [1877, 1881]: Minsk province. Also Pont [1986b, 2013].

MATERIAL: Vitebsk reg.: Berezinsky Biosphere Reserve, Kraitsy vill. $\left(\approx 54.67^{\circ} \mathrm{N} 28.27^{\circ} \mathrm{E}\right), 06 . \mathrm{VIII} .1969$, E. Antonova, 2 \%o (ZMUM).

\section{Thricops semicinereus (Wiedemann, 1817)}

Schnabl [1886, 1887a]: as Aricia semicinereus (Wiedemann, 1817), Minsk reg., Pukhovichy distr. (Minsk province), and Vitebsk reg., Liozno distr. (Mogilev province). Also Pont [1986b, 2013].

MATERIAL: Vitebsk reg.: Orsha distr., Dnieper R., $54.543^{\circ} \mathrm{N}$ $30.463^{\circ} \mathrm{E}, 11 . \mathrm{VI} .2017$, N. Vikhrev, $10^{7} 1$ 우 (ZMUM); Orsha distr., Svyatoe L., $54.686^{\circ} \mathrm{N} 30.442^{\circ} \mathrm{E}, 12$. VI.2017, N. Vikhrev, $10^{7}$ (ZMUM). 


\section{Thricops simplex (Wiedemann, 1817)}

MATERIAL: Brest reg.: Belovezhskaya Pushcha, $52.587^{\circ} \mathrm{N}$ 23.876 ${ }^{\circ}$ E, 17.VIII-06.X.2017, E. Makovetskaya, 1 우 (SPCB). Grodno reg.: Novogrudok distr., Svitiaziansky reserve, $53.430^{\circ} \mathrm{N}$ 25.884 ${ }^{\circ}$ E, 23.VII.2018, E. Makovetskaya, $10^{7}$ (SPCB).

\section{Drymeia hamata (Fallen, 1823)}

Schnabl [1877, 1881]: Minsk province. Also Pont [1986b, 2013].

\section{Drymeia vicana (Harris, 1780)}

Pont [1986b, 2013].

MATERIAL: Brest reg.: Belovezhskaya Pushcha Yasen' env. $\left(\approx 52.55^{\circ} \mathrm{N} 24.04^{\circ} \mathrm{E}\right)$, A.V. Birg, $10^{7}$ (SPCB). Vitebsk reg.: Dokshytsy distr., Severnoye Gnezdilovo env. $\left(\approx 54.82^{\circ} \mathrm{N} 27.75^{\circ} \mathrm{E}\right), 18$ 19.VI.1966, A.V. Birg, $10^{7}$ (SPCB) (misidentified as Hebecnema umbratica).

16. Hydrotaea aenescens (Wiedemann, 1830)

This species was reared by E. Makovetskaya from maggots, which were sold on fishermen's market in Zhdanovichy (Minsk), $20^{\top} \sigma^{\top} 2$ 우.

17. Hydrotaea cyrtoneurina (Zetterstedt, 1845)

MATERIAL: Vitebsk reg.: Orsha distr., Svyatoe L., $54.686^{\circ} \mathrm{N}$ 30.442 ${ }^{\circ}$ E, 12.VI.2017, N. Vikhrev, $10^{7}$ (ZMUM).

\section{Hydrotaea dentipes (Fabricius, 1805)}

Birg [1971]: widespread. Also Schnabl [1877, 1881], Erofeeva et al. [1960] and Pont [1986b, 2013].

MATERIAL: Grodno reg.: Novogrudok distr., Svitiaziansky reserve, $53.430^{\circ} \mathrm{N} 25.884^{\circ} \mathrm{E}, 23 . \mathrm{VII} .2018$, E. Makovetskaya, $10^{\prime}$ (SPCB). Minsk reg.: Volozhin distr., Nalybokskaya Pushcha, Kozliki vill., $53.904^{\circ} \mathrm{N} 26.422^{\circ} \mathrm{E}, 06 . \mathrm{V} .2017$, E. Makovetskaya, $10^{\top}$ (SPCB). Vitebsk reg.: Senno distr., Kruglyany env. $\left(\approx 54.89^{\circ} \mathrm{N}\right.$ $\left.29.98^{\circ} \mathrm{E}\right), 25-26 . V .1966$, A.V. Birg, $10^{7}$ (SPCB).

\section{Hydrotaea diabolus (Harris, 1780)}

Schnabl [1877, 1881]: as Hydrotaea ciliata (Fabricius, 1794), Minsk province. Also Pont [1986b, 2013].

\section{Hydrotaea ignava (Harris, 1780)}

As Ophyra leucostoma (Wiedemann, 1817) Schnabl [1877, 1881]: Minsk province; Erofeeva et al. [1960]: Minsk; Birg [1971]: Belovezhskaya Pushcha. Also Pont [1986b, 2013].

\section{Hydrotaea irritans (Fallen, 1823)}

MATERIAL: Minsk reg.: Minsk, Central Botanical Garden, $53.911^{\circ} \mathrm{N} 27.619^{\circ} \mathrm{E}, 26$. VII.2017, E. Makovetskaya, $10^{7}$ (SPCB); Minsk distr., near Volchkovichy vill., $53.809^{\circ} \mathrm{N} 27.384^{\circ} \mathrm{E}$, 17.VII.2017, E. Makovetskaya, $10^{7}$ (SPCB).

\section{Hydrotaea meridionalis Portschinsky, 1882}

Schnabl [1877, 1881]: as Hydrotaea curvipes (Fallen, 1825), Minsk province. Also Pont [1986b, 2013].

\section{Hydrotaea meteorica (Linnaeus, 1758)}

Schnabl [1877, 1881]: Minsk province. Also Pont [1986b, 2013] and Nakhaenko \& Kaplich [2007].

\section{Hydrotaea pandellei Stein, 1899}

MATERIAL: Vitebsk reg.: Orsha distr., Svyatoe L., $54.686^{\circ} \mathrm{N}$ 30.442 ${ }^{\circ}$ E, 12.VI.2017, N. Vikhrev, $10^{\top}$ (ZMUM).

25. Hydrotaea pellucens Portschinsky, 1879

Portschinsky [1880]: Mogilev province, Mogilev env., type locality. Also Pont [1986b, 2013], Sorokina \& Pont [2010] and Vikhrev [2013].
26. Hydrotaea velutina Robineau-Desvoidy, 1830 Birg [1971]: Belovezhskaya Pushcha.

27. Potamia littoralis Robineau-Desvoidy, 1830

Birg [1971]: as Dendrophaonia querceti (Bouche,1834), Belovezhskaya Pushcha. Also Gembicky [1968].

28. Mesembrina meridiana (Linnaeus, 1758)

Schnabl [1877, 1881]: Minsk province; Portschinsky [1874]: Mogilev province; Arnold [1901]: Mogilev reg., Gorki distr.; Birg [1971]: Vitebsk reg., Vitebsk, Polotsk and Dokshytsy districts. Also Pont [1986b, 2013].

MATERIAL: Brest reg.: Luninets distr., near Lunin, $52.317^{\circ} \mathrm{N}$ 26.618 ${ }^{\circ}$ E, 19.VIII.2017, E. Makovetskaya, 1 우 (SPCB). Minsk reg.: Volozhin distr., Nalybokskaya Pushcha, Belokorets vill., $54.018^{\circ} \mathrm{N}$ $26.509^{\circ}$ E, 17.IX.2017, E. Makovetskaya, 1 ( (SPCB). Vitebsk reg.: Vitebsk distr., near Borovlyany $\left(\approx 55.31^{\circ} \mathrm{N} 30.11^{\circ} \mathrm{E}\right), 31 . \mathrm{VII} .1966$, A.V. Birg, 1 + (SPCB).

\section{Mesembrina mystacea (Linnaeus, 1758)}

Schnabl [1877, 1881]: Minsk province; Portschinsky [1874]: Mogilev province; Arnold [1901]: Mogilev reg., Gorki distr. Also Pont [1986b, 2013].

30. Mesembrina resplendens Wahlberg, 1844

Portschinsky [1874, 1892]: Vitebsk reg., Orsha distr. Also Pont [1986b, 2013].

\section{Polietes domitor (Harris, 1780)}

Birg [1971]: as Polietes albolineata (Fallen, 1823), Minsk and Minsk reg., Myadzyel distr.

MATERIAL: Minsk reg.: Myadzyel distr., Lotva env. $\left(\approx 54.98^{\circ} \mathrm{N}\right.$ 26.89 ${ }^{\circ}$ E), 22-23.VII.1965, A.V. Birg, 1 우 (SPCB).

\section{Polietes lardarius (Fabricius, 1781)}

Draber-Monko [1995]: Berezinsky Biosphere Reserve. MATERIAL: Brest reg.: Belovezhskaya Pushcha, $52.573^{\circ} \mathrm{N}$ $23.802^{\circ}$ E, 06.X.2017, E. Makovetskaya, 19 (SPCB). Grodno reg.: Novogrudok distr., Svitiaziansky reserve, $53.430^{\circ} \mathrm{N} 25.884^{\circ} \mathrm{E}$, 23.VII.2018, E. Makovetskaya, $10^{7} 2$ 우 (SPCB); Valkovysk town, $53.112^{\circ} \mathrm{N} 24.404^{\circ} \mathrm{E}, 13 . \mathrm{IX} .2017$, E. Makovetskaya, 1 우 (SPCB). Minsk reg.: Lahoysk distr., Malinovka vill., $54.466^{\circ} \mathrm{N} 27.961^{\circ} \mathrm{E}$, 18.VI.2017, E. Makovetskaya, 2우 (SPCB); Stolbcy distr., $53.508^{\circ} \mathrm{N} 26.665^{\circ} \mathrm{E}, 10 . \mathrm{VI} .2018$, E. Makovetskaya, $4 \bigcirc^{7} \mathrm{O}^{\top} 3$ 우 (SPCB).

\section{Musca autumnalis De Geer, 1776}

Birg [1971]: widespread. Also Schnabl [1877, 1881], Erofeeva et al. [1960], Pont [1986b, 2013], Draber-Monko [1995], Nakhaenko \& Kaplich [2007].

MATERIAL: Grodno reg.:, Karelichy distr., near Siniavskaya Sloboda $53.593^{\circ} \mathrm{N} 26.337^{\circ} \mathrm{N} 27$. VII.2018, E. Makovetskaya, $10^{7}$ (SPCB). Minsk reg.: Stolbcy distr., near Semenchitsy, $53.545^{\circ} \mathrm{N}$ $26.699^{\circ}$ E, 20.VIII.2018, E. Makovetskaya, 2 우 (SPCB); Stolbcy distr., Nalybokskaya Pushcha, $53.850^{\circ} \mathrm{N} 26.426^{\circ} \mathrm{E}, 05 . \mathrm{V} .2018$, E. Makovetskaya, 19 (SPCB). Vitebsk reg.: Berezinsky Biosphere Reserve, Stvol'no vill., $54.766^{\circ} \mathrm{N} 28.321^{\circ} \mathrm{E}, 29$. VIII.2017, E. Makovetskaya, $10^{7}$ (SPCB).

\section{Musca domestica Linnaeus, 1758}

Birg [1971]: widespread. Also Salaview [1930], Danilevich [1958], Erofeeva et al. [1960], Gembicky [1968], Nakhaenko \& Kaplich [2007], Gorovenko [2014].

MATERIAL: Minsk reg.: Stolbcy distr., near Semenchitsy, $53.545^{\circ} \mathrm{N} 26.699^{\circ} \mathrm{E}, 20 . \mathrm{VIII} .2018$, E. Makovetskaya, 1 우 (SPCB).

35. Musca larvipara Portschinsky, 1910

Birg [1971]: widespread. Also Erofeeva et al. [1960]. 
36. Musca tempestiva Fallen, 1817

Nakhaenko \& Kaplich [2007].

37. Morellia hortorum (Fallen, 1817)

Salaview [1930]: Mogilev reg., Gorki distr.

38. Morellia simplex (Loew, 1857)

Birg [1971]: Belovezhskaya Pushcha and Vitebsk reg., Dokshytsy distr. Also Nakhaenko \& Kaplich [2007].

\section{Neomyia cornicina (Fabricius, 1781)}

Birg [1971]: as Orthellia (Cryptolucilia) caesarion Meigen, 1838, Minsk and Vitebsk reg., Dokshytsy, Hlybokaye and Senno districts; Draber-Monko [1995]: Berezinsky Biosphere Reserve.

MATERIAL: Brest reg.: Belovezhskaya Pushcha, $52.573^{\circ} \mathrm{N}$ $23.802^{\circ}$ E, 6.X.2017, E. Makovetskaya, $40^{7} \mathrm{O}^{\top} 3$ 웅 (SPCB). Minsk reg.: Minsk, Central Botanical Garden, $53.911^{\circ} \mathrm{N} 27.619^{\circ} \mathrm{E}$, IIIIV.2017, E. Makovetskaya, $10^{7}$ (SPCB).

40. Neomyia viridescens (Robineau-Desvoidy, 1830)

MATERIAL: Gomel reg.: Brahin distr., $51.5195^{\circ} \mathrm{N} 30.5135^{\circ} \mathrm{E}$ 20.V.2018, E. Makovetskaya, 19 (SPCB). Vitebsk reg.: Senno distr., Konstantovo env. $\left(\approx 54.85^{\circ} \mathrm{N} 30.03^{\circ} \mathrm{E}\right), 30-31 . V I I .1966$, A.V. Birg, 1 ( (SPCB) (misidentified by Birg as N. cornicina).

\section{Pyrellia rapax (Harris, 1780)}

Schnabl [1877, 1881]: as Homalomyia serena (Meigen, 1826), Minsk provinince; Also Birg [1970] as Pyrellia serena (Meigen, 1826).

42. Pyrellia vivida Robineau-Desvoidy, 1830

As Pyrellia cadaverina (Linnaeus, 1758) Schnabl [1877, 1881]: Minsk province; Birg [1971]: Minsk env. and Minsk reg.,Valozhyn distr.; Draber-Monko [1995]: Berezinsky Biosphere Reserve. Also Pont [1986b, 2013].

\section{Eudasyphora cyanicolor (Zetterstedt, 1845)}

Draber-Monko [1995]: Berezinsky Biosphere Reserve.

MATERIAL: Brest reg.: Bereza distr., Sporovsky reserve, near Mostyki, $52.415^{\circ} \mathrm{N} 25.096^{\circ} \mathrm{E}, 16-30 . \mathrm{VII} .2017$, E. Makovetskaya, 3 오 (SPCB); Bereza distr., Sporovsky reserve, near Strigin, $52.482^{\circ} \mathrm{N}$ $25.034^{\circ} \mathrm{E}, 16-29 . \mathrm{VII} .2017$, E. Makovetskaya, 2 우 (SPCB); Liakhavichy distr., Rusinovichy env., $52.976^{\circ} \mathrm{N} 26.319^{\circ} \mathrm{E}, 10 . \mathrm{VI} .2017$, E. Makovetskaya, 1 우 (SPCB). Gomel reg.: Pripyatsky National Park, $52.050^{\circ} \mathrm{N} 28.166^{\circ} \mathrm{E}, 17 . \mathrm{V}-08 . \mathrm{VI} .2018$, E. Makovetskaya, $10^{7} 2$ 우워 (SPCB). Grodno reg.: Novogrudok distr., Svitiaziansky reserve, $53.430^{\circ} \mathrm{N} 25.884^{\circ} \mathrm{E}, 23 . \mathrm{VII} .2018$, E. Makovetskaya, 19 (SPCB) Slonim distr., Slonimsky reserve, $53.099^{\circ} \mathrm{N} 25.541^{\circ} \mathrm{E}$, 13.IV.2018, E. Makovetskaya, 10우 (SPCB); Volkovysk distr., Zamkovy Les reserve $53.190^{\circ} \mathrm{N} 25.884^{\circ} \mathrm{E}, 15 . \mathrm{IV} .2018$, E. Makovetskaya, $20^{\top} \mathrm{O}^{\top} 1$ 1 (SPCB). Minsk reg.: Stolbcy distr., $53.508^{\circ} \mathrm{N} 26.665^{\circ} \mathrm{E}, 10 . \mathrm{VI} .2018$, E. Makovetskaya, 2 OP (SPCB); Stolbcy distr., Nalybokskaya Pushcha, $53.850^{\circ} \mathrm{N} 26.426^{\circ} \mathrm{E}, 05$. V.2018, E. Makovetskaya, 2 우 (SPCB).

\section{Stomoxys calcitrans (Linnaeus, 1758)}

Arnold [1901]: Mogilev province; Salaview [1930]: Mogilev reg., Gorki distr.; Erofeeva et al. [1960]: Minsk; Birg [1971]: Minsk, Vitebsk reg. and Belovezhskaya Pushcha. Also Nakhaenko \& Kaplich [2007].

MATERIAL: Brest reg.: Liakhavichy distr., Rusinovichy env., $52.976^{\circ} \mathrm{N} 26.319^{\circ} \mathrm{E}, 03 . X I I .2016$, E. Makovetskaya, 1 을 (SPCB) Pruzhany distr., Shani vill., $52.519^{\circ} \mathrm{N} 24.401^{\circ} \mathrm{E}, 15 . \mathrm{VII} .2017$, E. Makovetskaya, 1 우 (SPCB).

\section{Haematobosca stimulans (Meigen, 1824)}

As Haemathobia stimulans (Meigen, 1824) Nakhaenko \& Kaplich [2007]; Gorovenko [2014]: Vitebsk reg.
Subfamily Phaoniinae

46. Phaonia angelicae (Scopoli, 1763)

MATERIAL: Minsk reg.: Lahoysk distr., Kupalovsky reserve, $54.228^{\circ} \mathrm{N} 27.488^{\circ} \mathrm{E}, 27 . V^{2} .2018$, E. Makovetskaya, $10^{\top 7}$ (SPCB).

\section{Phaonia angulicornis (Zetterstedt, 1838)}

Pont [1986b, 2013].

48. Phaonia consobrina (Zetterstedt, 1838)

Schnabl [1877, 1881]: as Aricia marmorata Zetterstedt, 1860, Minsk province.

\section{Phaonia errans (Meigen, 1826)}

Schnabl [1877, 1881]: as Aricia erratica (Fallen, 1825) and $A$. errans (Meigen, 1826), Minsk province. Also Pont [1986b, 2013].

\section{Phaonia falleni Michelsen, 1977}

Schnabl [1877, 1881, 1886, 1887a, b]: as Aricia vagans (Fallen, 1825), Minsk province, Mogilev province (Vitebsk reg., Liozno distr.) and Polesie. Also Pont [2013].

\section{Phaonia fuscata (Fallen, 1825)}

Schnabl [1877, 1881, 1886, 1887a, 1888]: as Spilogaster fuscata (Fallen, 1825) or as Aricia fuscata (Fallen, 1825), Minsk province and Vitebsk reg., Liozno distr. (Mogilev prov.). Also Pont [1986b, 2013].

\section{Phaonia incana (Wiedemann, 1817)}

Schnabl [1877, 1881, 1886, 1887a, 1888]: as Aricia incana (Wiedemann, 1817), Minsk province. Also Pont [1986b, 2013].

MATERIAL: Vitebsk reg.: Berezinsky Biosphere Reserve, Kraitsy vill. $\left(54.67^{\circ} \mathrm{N} 28.27^{\circ} \mathrm{E}\right), 12 . \mathrm{VIII} .1969$, E. Antonova, $10^{7} 2$ 우 (ZMUM).

\section{Phaonia kowarzii Schnabl, 1887}

Schnabl [1886, 1887a]: as Aricia kowarzii, Vitebsk reg., Liozno distr., Zachernya vill. $\left(\approx 54.92^{\circ} \mathrm{N} 30.77^{\circ} \mathrm{E}\right)$, type locality. Also Pont [1986b, 2013], Sorokina \& Pont [2010], Vikhrev \& Erofeeva [2018].

\section{Phaonia laeta (Fallen, 1823)}

Schnabl [1877, 1881, 1886, 1887a]: as Aricia laeta (Fallen, 1823), Minsk province. Also Pont [2013].

\section{Phaonia latipalpis Schnabl, 1911}

Schnabl in Schnabl et Dziedzicki [1911]: Mogilev province, type locality. Also Pont [1986b, 2013], Sorokina \& Pont [2010].

\section{Phaonia meigeni Pont, 1986}

Schnabl [1886, 1887a]: as Aricia lugubris (Meigen, 1826): Vitebsk reg., Liozno distr., and Mogilev reg., Krasnapolle distr. Also Pont [2013].

\section{Phaonia pallida (Fabricius, 1787)}

Schnabl [1886, 1887a]: as Aricia pallida (Fabricius, 1787), Vitebsk reg., Liozno distr. and Minsk reg., Barysaw env. and Pukhovichy distr.; Birg [1971]: Belovezhskaya Pushcha and Vitebsk reg., Dokshytsy distr. Also Pont [1986b, 2013], Vikhrev \& Erofeeva [2018].

MATERIAL: Brest reg.: Belovezhskaya Pushcha, $52.587^{\circ} \mathrm{N}$ 23.876 ${ }^{\circ}$ E, 17.VIII-06.X.2017, E. Makovetskaya, 2우우 (SPCB); Belovezhskaya Pushcha NR $\left(52.5^{\circ} \mathrm{N} 23.8^{\circ} \mathrm{E}\right), 17.07-7.08 .1961$, E. Narchuk, 7우우 (ZIN); Bereza distr., Sporovsky reserve, near 
Mostyki, 52.415 ${ }^{\circ}$ 25.096 ${ }^{\circ} \mathrm{E}, 16-30$. VII.2017, E. Makovetskaya, 4 우 (SPCB); Bereza distr., Sporovsky reserve, near Strigin, $52.482^{\circ} \mathrm{N} 25.034^{\circ} \mathrm{E}, 16-29$.VII.2017, E. Makovetskaya, $10^{\circ}$ (SPCB); border of Kobryn and Pruzhany districts, near Oranchitsy vill., $52.398^{\circ} \mathrm{N} 24.565^{\circ} \mathrm{E}, 16-29$. VII.2017, E. Makovetskaya, 1 \% (SPCB). Minsk reg.: Stolbcy distr., near Opechki vill., $53.512^{\circ} \mathrm{N}$ 26.649 ${ }^{\circ}$ E, 21.VII.2018, E. Makovetskaya, E. Setrakova, 200 (SPCB). Vitebsk reg.: Dokshytsy distr., Severnoye Gnezdilovo env. ( $\left.\approx 54.82^{\circ} \mathrm{N} 27.75^{\circ} \mathrm{E}\right), 18-19$. VI.1966, A.V. Birg, $10^{7}$ (SPCB).

\section{Phaonia perdita (Meigen, 1830)}

Schnabl [1877, 1881]: as Aricia perdita (Meigen, 1830), Minsk province. Also Pont [1986b, 2013].

59. Phaonia pratensis (Robineau-Desvoidy, 1830) Pont [2013].

\section{Phaonia rufiventris (Scopoli, 1763)}

Schnabl [1886, 1887a]: as Aricia scutellaris (Fallen, 1825), Vitebsk reg., Liozno distr. and Minsk reg., Pukhovichy distr.; Birg [1971]: misidentification; Vikhrev \& Erofeeva [2018]: Brest reg., Belovezhskaya Pushcha. Also Pont [1986b, 2013].

MATERIAL: Brest reg.: Belovezhskaya Pushcha, $52.587^{\circ} \mathrm{N}$ $23.876^{\circ}$ E, 17.VIII-6.X.2017, E. Makovetskaya, 1 우 (SPCB).

\section{Phaonia serva (Meigen, 1826)}

Schnabl [1877, 1881, 1886, 1887a]: Minsk province and Vitebsk reg., Liozno distr., Mogilev reg., Krasnapolle distr. Also Pont [1986b, 2013].

62. Phaonia siebecki Schnabl in Schnabl et Dziedzicki, 1911

MATERIAL: Vitebsk reg.: Orsha distr., Svyatoe L., $54.686^{\circ} \mathrm{N}$ $30.442^{\circ} \mathrm{E}, 12$. VI.2017, N. Vikhrev, $20^{7} \sigma^{7}$ (ZMUM).

\section{Phaonia subventa (Harris, 1780)}

Vikhrev \& Erofeeva [2018]: Vitebsk reg., Orsha distr. MATERIAL: Brest reg.: Bereza distr., Sporovsky reserve, near Mostyki, $52.415^{\circ} \mathrm{N} 25.096^{\circ} \mathrm{E}, 16-30 . \mathrm{VII} .2017$, E. Makovetskaya, $30^{7} 0^{7} 4$ 웅 (SPCB). Vitebsk reg.: Orsha distr., Dnieper R., $54.543^{\circ} \mathrm{N}$ $30.463^{\circ} \mathrm{E}, 11 . \mathrm{VI} .2017$, N. Vikhrev, 2 우 (ZMUM); Dokshytsy distr., Severnoye Gnezdilovo env. $\left(\approx 54.82^{\circ} \mathrm{N} 27.75^{\circ} \mathrm{E}\right), 18-19$. VI.1966, A.V. Birg, $10^{7}$ (SPCB, misidentified by Birg as P. scutellaris $=$ P. rufiventris).

\section{Phaonia tuguriorum (Scopoli, 1763)}

Schnabl [1886, 1887a, 1888]: as Aricia signata (Meigen, 1826) and as Aricia errans var. polonica Schnabl, 1888, Minsk province. Also Pont [1986b, 2013].

MATERIAL: Minsk reg.: Minsk, Kurchatova str., protected area "Dubrava", $53.834^{\circ} \mathrm{N} 27.469^{\circ} \mathrm{E}, 13 . X .2018$, E. Makovetskaya, $10^{7}$ (SPCB).

\section{Phaonia valida (Harris, 1780)}

Pont [1986b; 2013].

MATERIAL: Brest reg.: Bereza distr., Sporovsky reserve, near Mostyki, $52.415^{\circ} \mathrm{N} 25.096^{\circ} \mathrm{E}, 30 . \mathrm{VII}-19 . \mathrm{VIII} .2017$, E. Makovetskaya, 1 우 (SPCB). Grodno reg.: Novogrudok distr., Svitiaziansky reserve, $53.430^{\circ} \mathrm{N} 25.884^{\circ} \mathrm{E}, 23 . \mathrm{VII} .2018$, E. Makovetskaya, 5 우 (SPCB). Minsk reg.: Volozhin distr., Nalybokskaya Pushcha, Belokorets vill., $54.018^{\circ} \mathrm{N} 26.509^{\circ} \mathrm{E}, 17$. IX.2017, E. Makovetskaya, 3 우 (SPCB). Vitebsk reg.: Berezinsky Biosphere Reserve, Stvol'no vill., $54.766^{\circ} \mathrm{N} 28.321^{\circ} \mathrm{E}, 29$.VIII.2017, E. Makovetskaya, $10^{\circ}$ (SPCB) and Domzheritsy env., $54.772^{\circ} \mathrm{N} 28.303^{\circ} \mathrm{E}$, E. Makovetskaya, $10^{7}(\mathrm{SPCB})$.

\section{Helina allotalla (Meigen, 1830)}

Pont [1986b; 2013].
67. Helina annosa (Zetterstedt, 1838)

MATERIAL: Vitebsk reg.: Orsha distr., Dnieper R., $54.543^{\circ} \mathrm{N}$ $30.463^{\circ}$ E, 11.VI.2017, N. Vikhrev, $10^{\top}$ (ZMUM) and Svyatoe L., $54.686^{\circ} \mathrm{N} 30.442^{\circ} \mathrm{E}, 12$. VI.2017, N. Vikhrev, $10^{7}$ (ZMUM).

\section{Helina ciliatocosta (Zetterstedt, 1845)}

Schnabl [1877, 1881]: as Coenosia ciliatocosta (Zetterstedt, 1845), Minsk province. Also Pont [1986b, 2013].

\section{Helina cilipes (Schnabl, 1902)}

Schnabl, Dziedzicki [1911]: as Enoplopteryx cilipes sp. n., Minsk province, type locality. Also Pont [1986b, 2013].

70. Helina depuncta (Fallen, 1825)

Birg [1971]: Belovezhskaya Pushcha.

MATERIAL: Minsk reg.: Minsk, Kurchatova str., protected area "Dubrava", $53.834^{\circ} \mathrm{N} 27.469^{\circ} \mathrm{E}, 13 . X .2018$, E. Makovetskaya, 1 우 (SPCB).

\section{Helina evecta Harris, 1780}

Schnabl [1887a, 1888]: as Aricia lucorum (Fallen, 1823) and as A. lucorum var. mohyleviensis Schnabl, 1888, Minsk province and Vitebsk reg., Liozno distr. (Mogilev prov.). Also Pont [1986b; 2013].

MATERIAL: Minsk reg.: Minsk, Central Botanical Garden, $53.916^{\circ} \mathrm{N} 27.609^{\circ} \mathrm{E}, 13 . \mathrm{IX} .2018$, E. Makovetskaya, 1 우 (SPCB).

72. Helina impuncta (Fallen, 1825)

Schnabl [1877, 1881]: as Spilogaster impuncta (Fallen, 1825), Minsk province. Also Pont [1986b, 2013].

MATERIAL: Vitebsk reg.: Berezinsky Biosphere Reserve, Kraitsy vill. $\left(\approx 54.67^{\circ} \mathrm{N} 28.27^{\circ} \mathrm{E}\right)$, 03.IX.1969, E. Antonova, 1 ( (ZMUM); Orsha distr., Svyatoe L., $54.686^{\circ}$ N $30.442^{\circ}$ E, 12.VI.2017, N. Vikhrev, 1 우 (ZMUM).

73. Helina latitarsis Ringdahl, 1924

Pont [2013].

74. Helina obscurata (Meigen, 1826)

MATERIAL: Minsk reg.: Barysaw distr., Vialikaje Stachava env., 54.149 $\mathrm{N} 28.636^{\circ} \mathrm{E}$, 07.VII.2013, D. Gavryushin, $10^{7}$ (ZMUM).

75. Helina obscuratoides (Schnabl, 1887)

Schnabl [1886, 1887a, 1888]: as Aricia obscuratoides from Vitebsk reg., Liozno distr., type locality. Also Pont [1986b, 2013] and Sorokina \& Pont [2010].

76. Helina protuberans (Zetterstedt, 1845)

Pont [2013].

77. Helina quadrum (Fabricius, 1805)

Schnabl [1877, 1881]: as Spilogaster quadrum (Fabricius, 1805), Minsk province. Also Pont [1986b, 2013].

MATERIAL: Vitebsk reg.: Berezinsky Biosphere Reserve, Kraitsy vill. $\left(\approx 54.67^{\circ} \mathrm{N} 28.27^{\circ} \mathrm{E}\right), 16$.VIII.1969, E. Antonova, 1 ㅇ (ZMUM).

78. Helina reversio (Harris, 1780)

Schnabl [1877, 1881]: as Spilogaster duplicata (Meigen, 1826), Minsk province. Also Pont [1986b, 2013].

MATERIAL: Minsk reg.: Minsk, Central Botanical Garden, $53.916^{\circ} \mathrm{N} 27.603^{\circ} \mathrm{E}, 21 . \mathrm{IX} .2018$, E. Makovetskaya, 1 ㅇ (SPCB).

\section{Helina sexmaculata (Preyssler, 1791)}

Schnabl [1877, 1881]: as Spilogaster uliginosa (Fallen, 1825), Minsk province. Also Pont [1986b, 2013].

80. Helina subvittata (Seguy, 1923)

Pont [2013]. 
Subfamily Mydaeinae

81. Mydaea corni (Scopoli, 1763)

Birg [1971]: as Mydaea pagana (Fabricius, 1794), Minsk reg., Valozhyn distr. and Vitebsk reg., Dokshytsy distr.

MATERIAL: Minsk reg.: Volozhin distr., Kapustino env. $\left(\approx 54.06^{\circ} \mathrm{N} 26.55^{\circ} \mathrm{E}\right), 14 . \mathrm{VII} .1965, \mathrm{~A} . \mathrm{V}$. Birg, $10^{7}$ (SPCB).

82. Mydaea humeralis Robineau-Desvoidy, 1830

MATERIAL: Vitebsk reg.: Orsha distr., Svyatoe L., $54.686^{\circ} \mathrm{N}$ 30.442 ${ }^{\circ}$ E, 12.VI.2017, N. Vikhrev, $10^{7}$ (ZMUM).

\section{Mydaea urbana (Meigen, 1826)}

Schnabl [1877, 1881]: as Aricia urbana (Meigen, 1826), Minsk province. Also Pont [1986b, 2013].

MATERIAL: Grodno reg.: Novogrudok distr., Svitiaziansky reserve, $53.430^{\circ} \mathrm{N} 25.884^{\circ} \mathrm{E}, 23 . \mathrm{VII} .2018$, E. Makovetskaya, 5 우 (SPCB). Minsk reg.: Minsk, Soltysa str., $53.896^{\circ} \mathrm{N} 27.657^{\circ} \mathrm{E}$ 28.VI.2017, E. Makovetskaya, 3 우 (SPCB). Vitebsk reg.: Berezinsky Biosphere Reserve, Stvol'no vill., $54.766^{\circ} \mathrm{N} 28.321^{\circ} \mathrm{E}$, 29.VIII.2017, E. Makovetskaya, 1 우 (SPCB).

84. Myospila meditabunda (Fabricius, 1781)

Erofeeva et al. [1960]: Minsk; Birg [1971]: Belovezhskaya Pushcha and Minsk.

85. Hebecnema umbratica (Meigen, 1826)

Schnabl [1886, 1887a, 1888]: as Aricia umbratica (Meigen, 1826), Mogilev province and Minsk reg., Pukhovichy distr.; Birg [1971]: misidentification. Also Pont [1986b, 2013].

86. Hebecnema vespertina (Fallen, 1823)

MATERIAL: Minsk reg.: Barysaw distr., Vialikaje Stachava env., 54.149 ${ }^{\circ} \mathrm{N} 28.636^{\circ} \mathrm{E}, 07$. VII.2013, D. Gavryushin, $10^{\top}$ (ZMUM).

87. Graphomya maculata (Scopoli, 1763)

Schnabl [1877, 1881]: Minsk province; Erofeeva et al. [1960]: Minsk; Birg [1971]: Minsk and Belovezhskaya Pushcha. Also Pont [1986b, 2013], including as Graphomya minor Robineau-Desvoidy, 1830.

MATERIAL: Brest reg.: Bereza distr., Sporovsky reserve, near Mostyki, $52.415^{\circ} \mathrm{N} 25.096^{\circ} \mathrm{E}, 16-30 . \mathrm{VII} .2017$, E. Makovetskaya, 1 우 (SPCB); Belovezhskaya Pushcha, Yasen' env. $\left(\approx 52.55^{\circ} \mathrm{N}\right.$ $\left.24.04^{\circ} \mathrm{E}\right)$, A.V. Birg, $10^{7} 2$ 우 (SPCB). Grodno reg.: Byerastavitsa distr., Svislochsky reserve, $53.425^{\circ} \mathrm{N} 23.942^{\circ} \mathrm{E}, 13 . \mathrm{VIII} .2017$, E. Makovetskaya, $1+$ (SPCB). Minsk reg.: Stolbcy distr., near Semenchitsy, $53.545^{\circ} \mathrm{N} 26.699^{\circ} \mathrm{E}, 20 . \mathrm{VIII} .2018$, E. Makovetskaya, $10^{\top} 1$ 우 (SPCB).

\section{Subfamily Coenosiinae}

88. Spilogona dispar (Fallen, 1823)

Schnabl [1886, 1887a]: as Aricia dispar (Fallen, 1823), Minsk province. Also Pont [1986b, 2013].

89. Lispe consanguinea Loew, 1858

Schnabl [1877, 1881]: Minsk province; Birg [1971]: Brest, Minsk and Minsk reg., Myadzyel distr.; Vikhrev [2014]: Minsk reg. Also Pont [1986b, 2013].

90. Lispe longicollis Meigen, 1826

Vikhrev [2014]: Minsk reg., Barysaw env.

91. Lispe melaleuca Loew, 1847

Vikhrev [2015b]: Minsk reg., Barysaw env.

92. Lispe nana Macquart, 1835

Vikhrev [2014]: Minsk reg., Barysaw env.
93. Lispe pygmaea Fallen, 1825

Schnabl [1877, 1881]: as Lispe simplicissima Loew, 1847, Minsk province. Also Pont [1986b, 2013].

94. Lispe superciliosa Loew, 1861

Vikhrev [2015b]: Minsk reg., Barysaw env.

95. Lispe tentaculata (De Geer, 1776) 2013].

Schnabl [1877, 1881]: Minsk province. Also Pont [1986b,

\section{Lispe uliginosa Fallen, 1825}

Schnabl [1877, 1881]: Minsk province; Vikhrev [2015b]: Minsk reg., Barysaw env.

97. Schoenomyza litorella (Fallen, 1823)

Schnabl [1877, 1881]: as Ochthiphila litorella (Fallen, 1823), Minsk province.

98. Coenosia agromyzina (Fallen, 1825)

MATERIAL: Minsk reg.: Minsk, Central Botanical Garden, $53.916^{\circ} \mathrm{N} 27.609^{\circ} \mathrm{E}, 13 . \mathrm{IX} .2018$, E. Makovetskaya, $2+9$ (SPCB).

99. Coenosia albicornis Meigen, 1826

Schnabl [1877, 1881]: Minsk province.

100. Coenosia bilineella (Zetterstedt, 1838)

Schnabl [1877, 1881]: Minsk province.

101. Coenosia campestris (Robineau-Desvoidy, 1830)

Schnabl [1877, 1881]: as Coenosia sexnotata Meigen, 1826, Minsk province.

102. Coenosia femoralis (Robineau-Desvoidy, 1830)

Schnabl [1877, 1881]: as Coenosia geniculata (Fallen, 1825), Minsk province. Also Pont [2013].

103. Coenosia intermedia (Fallen, 1825)

Schnabl [1877, 1881]: Minsk province. Also Pont [2013].

104. Coenosia mollicula (Fallen, 1825) 2013]

Schnabl [1877, 1881]: Minsk province. Also Pont [1986b,

MATERIAL: Minsk reg.: Barysaw env., $54.256^{\circ} \mathrm{N} 28.481^{\circ} \mathrm{E}$, 05.VII.2013, D. Gavryushin, $10^{7}$ (ZMUM).

105. Coenosia pedella (Fallen, 1825)

Schnabl [1877, 1881]: as Coenosia decipiens Meigen, 1826, Minsk province. Also Pont [1986b, 2013].

106. Coenosia pudorosa Collin, 1953

Schnabl \& Dziedzicki [1911]: as Coenosia luteicornis sp. n., Vitebsk reg., Liozno distr. Also Pont [1986b, 2013].

107. Coenosia pulicaria (Zetterstedt, 1845)

MATERIAL: Minsk reg.: Barysaw, Byarezina R., $54.239^{\circ} \mathrm{N}$ $28.494^{\circ}$ E, 05.VII.2013, D. Gavryushin, $10^{\text {T' }}$ (ZMUM).

108. Coenosia pumila (Fallen, 1825)

Pont [1986b, 2013].

MATERIAL: Minsk reg.: Barysaw, Byarezina R., $54.239^{\circ} \mathrm{N}$ $28.494^{\circ}$ E, 05.VII.2013, D. Gavryushin, $10^{7}$ (ZMUM).

109. Coenosia testacea (Robineau-Desvoidy, 1830)

Schnabl [1877, 1881]: as Coenosia tricolor Zetterstedt, 1845, Minsk province. Also Pont [2013]. 
MATERIAL: Minsk reg.: Barysaw env., $54.256^{\circ} \mathrm{N} 28.481^{\circ} \mathrm{E}$, 5.VII.2013, D. Gavryushin, $10^{\text {T }}$ (ZMUM).

110. Coenosia tigrina (Fabricius, 1775)

MATERIAL: Minsk reg.: Minsk, Central Botanical Garden, $53.916^{\circ} \mathrm{N} 27.603^{\circ} \mathrm{E}, 21 . I X .2018$, E. Makovetskaya, 1 우 (SPCB).

\section{Addendum}

List of species not found in Belarus but recorded from Bialowieza Primeval Forest (Polish part of Belovezhskaya Pushcha) by Draber-Monko [2001]:

Fannia latipalpis (Stein, 1892)

Fannia maniacata (Meigen, 1826)

Fannia parva (Stein, 1895)

Fannia postica (Stein, 1895)

Fannia tuberculata (Zetterstedt, 1849)

Thricops innocuus (Zetterstedt, 1838)

Thricops nigritellus (Zetterstedt, 1838)

Hydrotaea armipes (Fallen, 1825)

Hydrotaea pilitibia Stein, 1916

Morellia aenescens Robineau-Desvoidy, 1830

Phaonia halterata (Stein, 1898)

Phaonia lugubris (Meigen, 1826) as Ph. morio (Zettestedt, 1845)

Phaonia magnicornis (Zettestedt, 1845)

Phaonia mystica (Meigen, 1826)

Phaonia palpata (Stein, 1897)

Phaonia steinii (Strobl, 1898)

Helina abdominalis (Zettestedt, 1846)

Helina confinis (Fallen, 1825)

Helina parcepilosa (Stein, 1907) as H. calceataeformis (Scnabl in Schnabl et Dziedzicki, 1911)

Helina tetrastigma (Meigen, 1826)

Spilogona pacifica (Meigen, 1826)

Limnophora nigripes (Robineau-Desvoidy, 1830)

Lispocephala verna (Fabricius, 1794) as Caricea verna (Fabricius, 1794)

Macrorchis meditata (Fallen, 1825)

Coenosia nigridigita Rondani, 1866

Coenosia perpusilla Meigen, 1826

Coenosia trilineella (Zetterstedt, 1838)

ACKNOWLEDGEMENTS. We thank Anatoly Kulak (Minsk) for access to A. Birg collection; Olga Ovchinnikova and Galina Suleymanova (St-Petersburg) for access to ZIN collection. We thank Maria Yanbulat (Moscow) and Oleg Kosterin (Novosibirsk) for correcting our text. We are grateful to Ekaterina Setrakova; Yury Giginyak; Oleg Borodin (all Minsk) and Adrian Pont (Oxford) for various support of our work.

\section{References}

Arnold N. 1901. Catalogus insectirum provinciae Mohileviensis. St.-Petersburg. $150 \mathrm{pp}$.

Birg A.V. 1971. [Ecological and faunistic review of the flies from human settlements and surrounding areas in Byelorussia] // Belovezhskaya Pushcha. No.4. P.212-230 [in Russian].

Danilevich A. 1958. [Dependence of species composition and number of synanthropic flies on the place of breeding] // Tezisy mezhvuzovskoy nauchnoy konferenetsii po voprosam biologii i sel'skogo khozyaystva na temu: "Yedinstvo organizma i usloviy yego sushchestvovaniya (Organizm i sreda)". Moscow. P.25-26 [in Russian].
Draber-Monko A. 1995. Selected Calyptrata (Diptera) of the pine forests of the Berezinsky Biosphere Reserve in Byelorussia // Fragmenta Faunistica. Vol.38. No.7. P.165-179.

Draber-Monko A. 2001. Grupa Calyptrata// Gutowski G.M., Jaroszewicz B. (eds.) Catalogue of the fauna of Bialowieza Primeval Forest. Warszawa: Instytut Badawczy Lesnictwa. P.301-307.

Erofeeva T.V., Demianchenko R.P., Goldina G.S. Danilevich A.V. 1960. [Synanthropic flies of Minsk and methods of control them] // Trudy Tsentral'nogo nauchno-issledovatel'skogo infektsionnogo instituta. No.13. P.227-239 [in Russian].

Gembicky A.S. 1968. [The inhabitants of the nests of synanthropic birds of Byelorussia] // Tezisy dokladov tret'yey zoologicheskoy konferentsii, posvyashchennoy 50-letiyu obrazovaniya BSSR. Minsk. P.238-241 [in Russian].

Gorovenko M.V. 2014. [Transmission factors and prevention of helminth infections in the gastrointestinal tract of cattle in the northern zone of the Republic of Belarus] // Aktual'nyye problemy intensivnogo razvitiya zhivotnovodstva: materialy XVII Mezhdunarodnoy nauchno-prakticheskoy konferentsii, posvyashchennoy 80-letiyu kafedry zoogigieny, ekologii i mikrobiologii UO «BGSKHA». Gorki: Belarusian State Academy of Agriculture. P.57-62 [in Russian].

Nakhaenko A.G., Kaplich V.M. 2007. [About zoophilous flies of the north and central forest subzones of Belarus] // Materialy III Mezhdunarodnoy nauchno-prakticheskoy konferentsii "Sovremennyye ekologicheskiye problemy ustoychivogo razvitiya Polesskogo regiona i sopredel'nykh territoriy: nauka, obrazovaniye, kul'tura": in 3 parts. Part 1. Mozyr: Mozyr State Pedagogical University. P.183-184 [in Russian].

Neselovskaya V.K., Birg A.V., Goldina G.S. 1968. [Variances in the synanthropic flies number in Minsk] // Tezisy dokladov tret'yey zoologicheskoy konferentsii, posvyashchennoy 50-letiyu obrazovaniya BSSR. Minsk. P.194-195 [in Russian].

Pont A. 1986a. Fanniidae // Soos. A., Papp. A. (Eds). Catalogue of Palaearctic Diptera. Vol. 11. Scathophagidae - Hypodermatidae. Budapest: Akademiai Kiado. P.41-57.

Pont A. 1986b. Muscidae // Soos. A., Papp. A. (Eds). Catalogue of Palaearctic Diptera. Vol. 11. Scathophagidae - Hypodermatidae. Budapest: Akademiai Kiado: P.57-215.

Pont A.C. 2013. Fauna Europaea: Fanniidae \& Muscidae//Bystrowski C., Richter V.A., Pape T.P. 2013. Fauna Europaea: Diptera: Brachycera. Fauna Europaea version 2018.12, https://faunaeu.org.

Portschinsky I.A. 1874. [A monograph of the species of the genus Mesembrina that are found in the Russian Empire] // Trudy Russkogo entomologicheskogo obshchestva. T.7. P.55-60 [in Russian].

Portschinsky J. 1880. Diptera nova rossica et sibirica // Horae societatis entomologicae rossicae. T.15. P.157-158.

Portschinsky I. 1892. [Researches of dipteran insects of Russia. Part 1] // Horae societatis entomologicae rossicae. T.26. P.63-131 [in Russian].

Rozkošný R., Gregor F., Pont A.C. 1997. The European Fanniidae (Diptera) // Acta scientiarum naturalium Academiae scientiarum Bohemicae, Brno. Vol.31. P.1-80.

Salaview P. 1930. [Regular phenological observations in Gorki] // Annalen der Weissrussischen Staatlichen Akademie. Bd.11. S.91-102 [in Belarussian].

Schnabl J. 1877. [List of dipteran insects (Diptera) collected by J. Schnabl, Henr. Dziedzicki, J. Vankovich and Anders in various localities of the Polish Kingdom and Minsk Province] // Trudy pyatogo s'yezda russkikh yestestvoispytateley i vrachey v Varshave, Vyp. 3. Otdeleniye zoologii i sravnitel'noy anatomii. Warsaw. S.387-408 [in Russian].

Schnabl J. 1881. Spis Owadow dwuskrzydlych (Diptera) zebranych w Krolestwie Polskiem i gubernii Minskiej // Pamietnik fizyjograficzny. T.1. P.357-390.

Schnabl J. 1886. Contibutions à la faune diptérologique // Horae societatis entomologicae rossicae. T.20. P.271-440.

Schnabl J. 1887a. Contibutions à la faune diptérologique. St. Petersburg. $226 \mathrm{pp}$.

Schnabl J. 1887b. Aricia vagans Fall. (nec Schiner) // Horae societatis entomologicae rossicae. T.21. P.458-466. 
Schnabl J. 1888. Contibutions à la faune diptérologique. Additions aux descriptions precendentes des Arcica et descriprions des especes nouvelles // Horae societatis entomologicae rossicae. T.22. P.378-486.

Schnabl J., Dziedzicki H. 1911. Die Anthomyiden // Nova Acta Abhandlungen der Kaiserlichen Leopoldinisch-Carolinischen Deutschen Akademie de Naturforscher. Bd.95. Nr.2. S.53-358.

Sorokina V.S., Pont A. C. 2010. An annotated catalogue of the Muscidae (Diptera) of Siberia // Zootaxa. No.2597. P.1-87.

Vikhrev N.E. 2014. Taxonomic notes on Lispe (Diptera, Muscidae) Parts 1-9 // Amurian Zoological Journal. Vol.6. No.2. P.147-170.

Vikhrev N.E. 2015a. Review of the World fauna of the genus Azelia (Diptera, Muscidae) // Amurian Zoological Journal. Vol.7. No.1. P.33-42.
Vikhrev N.E. 2015b. Taxonomic notes on Lispe (Diptera, Muscidae). Parts 10-12 // Amurian Zoological Journal. Vol.7. No.3. P.228-247.

Vikhrev N.E. 2018. New species of Fannia Robineau-Desvoidy, 1830 (Diptera: Fanniidae) from Nepal // Russian Entomological Journal. Vol.27. No.2. P.207-209.

Vikhrev N.E., Erofeeva E.A. 2018. Review of the Phaonia pallida group (Diptera: Muscidae) // Russian Entomological Journal. No.27. No.3. P.315-322.

Wang M.F., Dong Y., Ao H. 2013. A review of the metallipennisgroup and fuscinata-group of Fannia Robineau-Desvoidy (Diptera: Fanniidae) // Annales de la Societe Entomologique de France. Vol.47. Nos3-4. P.487-500. 